\section{Produktpalette mit Zukunftspotenzial}

E nde November 2010 hat das Unternehmen HAL Allergy beim PaulEhrlich-Institut die Zulassungsunterlagen für das Subkutanpräparat Purethal ${ }^{\circledR}$ Milben sowie die Sublingualpräparate Sublivac ${ }^{\circledR}$ Fix Gräser, Bäume und Milben eingereicht. Damit sind fristgerecht die Anforderungen der Therapieallergene-Verordnung umgesetzt worden, denen zufolge bis zum 1. Dezember 2010 für noch nicht zugelassene Präparate zur spezifischen Immuntherapie mit den Allergenen von Gräsern, Frühblühern, Hausstaubmilben sowie Biene und Wespe ein entsprechender Antrag gestellt werden musste. Um die hierzu erforderlichen klinischen Daten zu Sicherheit und Wirksamkeit zu vervollständigen, wird eine Übergangsfrist von sieben Jahren gewährt.

Bereits zuvor hatte die Europäische Arzneibehörde die pädiatrischen Prüfpläne (PIP) für die genannten HALProdukte genehmigt. Damit erfüllen diese auch die Vorgaben der Verordnung Nr. 1901/2006 des Europäischen Parlaments, wonach mit dem Zulassungsantrag für ein neues Arzneimittel entweder die Ergebnisse pädiatrischer
Studien entsprechend einem gebilligten pädiatrischen Prüfkonzept vorgelegt werden müssen oder aber Belege dafür, dass eine Freistellung oder Zurückstellung gewährt wurde.

„Der deutsche Immuntherapiemarkt ist der größte Europas. Die Einreichung der Zulassungsunterlagen ist ein wichtiger Schritt und unterstreicht unsere Absicht, unsere Position zukünftig auszubauen", erklärte Harry Flore,

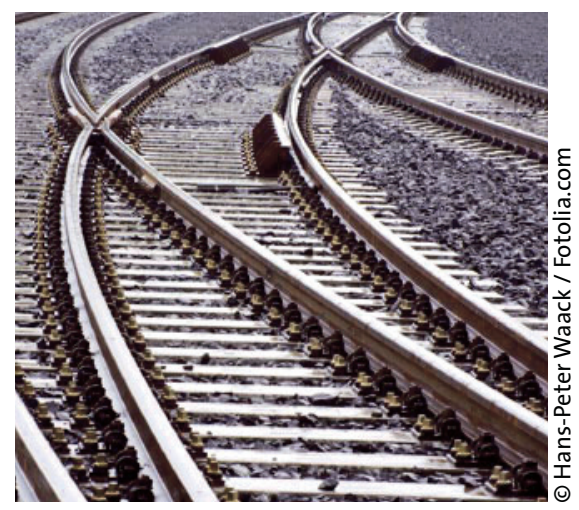

Das Unternehmen HAL hat jetzt die entscheidenden Weichen gestellt, um auch in Zukunft seine Präparate zur Hyposensibilisierung in Deutschland anbieten zu können.
Chief Executive Officer der niederländischen Unternehmensgruppe HAL Allergy. Indem diese sich verpflichte, ein klinisches Versuchsprogramm auf dem Stand der Wissenschaft durchzuführen, sichere sie die kontinuierliche Bereitstellung eines breiten Spektrums an Immuntherapeutika zur Behandlung inhalativer Allergien und bleibe zuverlässiger Partner für allergologisch tätige Ärzte und deren Patienten. Etliche Produkte haben die Ziellinie bereits durchschritten, wie der Geschäftsführer der Düsseldorfer Niederlassung, Jean Lumovici, bestätigte: „Glücklicherweise ist ein großer Teil unseres zeitgemäßen Produktportfolios wie Purethal ${ }^{\circledR}$ Gräser und Puretha ${ }^{\circledR}$ Bäume bereits heute zugelassen und wir können unsere Ressourcen auf die wenigen verbleibenden Projekte fokussieren.

Die Gruppe HAL Allergy wurde 1959 gegründet und ist einer der europaweit führenden Hersteller von Allergenextrakten für die Diagnostik und Therapie. Das Unternehmen beschäftigt heute über 280 Mitarbeiter, 60 davon bei der deutschen Tochter HAL Allergie GmbH mit Sitz in Düsseldorf.

Nach Informationen von HAL Allergie, Düsseldorf

Ein Präparat, das der Forderung nach dem Einsatz evidenzbasierter Therapieallergene bereits heute gerecht wird, ist Staloral ${ }^{300 ®}$ Birke. Ein Viertel der Befragten setzte das Präparat im letzten Jahr zu Behandlung einer Baumpollenallergie ein. Dabei begrüßten die Ärzte, dass das Präparat über eine Zulassung nach modernen Standards verfügt und in klinischen Studien evidenzbasierte Nachweise zur Wirksamkeit, Sicherheit und Verträglichkeit erbracht hat. Eine weitere Eigenschaft, die aus Sicht der Befragten für den Einsatz von Staloral ${ }^{300} \otimes$ Birke spricht, ist die Möglichkeit einer leitliniengerechten Behandlung sowie das einfache prä- bzw. cosaisonale Therapieschema.

Nach Informationen von Stallergenes, Kamp-Lintfort 